\title{
Similarity Invariant Delaunay Graph Matching
}

\author{
Dongjoe Shin and Tardi Tjahjadi \\ School of Engineering, University of Warwick, Coventry, UK, CV4 7AL \\ \{Dongjoe.Shin, T.Tjahjadi\} @warwick.ac.uk
}

\begin{abstract}
Delaunay tessellation describes a set of arbitrarily distributed points as unique triangular graphs which preserves most local point configuration called a clique regardless of noise addition and partial occlusion. In this paper, this structure is utilised in a matching method and proposed a clique-based Hausdorff Distance (HD) to address point pattern matching problems. Since the proposed distance exploits similarity invariant features extracted from a clique, it is invariant to rotation, translation and scaling. Furthermore, it inherits noise robustness from HD and has partial matching ability because matching performs on local entities. Experimental results show that the proposed method performs better than the existing variants of the general HD.
\end{abstract}

Keywords: Point pattern matching, Delaunay tessellation, Hausdorff distance, Similarity invariant distance.

\section{Introduction}

Point Pattern Matching (PPM) is a problem that searches the best point correspondences by investigating underlying point pattern and it includes many vision applications, e.g., motion estimation, image registration and object recognition [1,2, 3, 4. Although much research has been intensely done to address the problem, the PPM result is not reliable particularly when a point set includes noise or outliers, when whole or part of the data has been similarity transformed (e.g., rotated, translated and scaled) and when the number of matching points is different, i.e., some points should have either multiple matching opponents or none.

One classical approach to PPM is a spectral method that compares correlation of eigen vectors of a distance matrix referred to as a proximity matrix. The intra-distances between all possible pairs of points in an image are measured by a Gaussian-weighted distance metric and stored as a matrix in which eigen vectors are extracted as new features for matching 2]. Although the earlier methods show a weakness in dealing with partial matching, noise and significant image transformation, more recent methods address partial matching using a sub-matrix matching algorithm [5] and achieve robustness against noise by combining the spectral analysis with the Expectation Maximization (EM) framework [6]. Caelli et al. improve the accuracy of matching by re-normalising the eigen vectors and values used in comparison. The similarity of graph or tree of vertices is then measured by the distance of clusters in a tree or graph in the re-normalised subspace instead of the general point distance [7. 
Another approach is the Hausdorff Distance (HD) matching. The key advantage of using HD is that exact matching is not required when computing distance. Without a knowledge of exact point correspondences, HD measures distance between two sets of points. Moreover, it allows small perturbation and partial point pattern matching [8]. HD is a non-directional and nonlinear operation, and by making some changes in the distance function and combining two directional HDs, useful variants of HD can be generated, e.g., ranked HD and modified HD 9]. These methods have been extended to line and curve features in accurate recognition systems 10,11,13. A recent algorithm which associates an affine invariant coordinate called a homogeneous barycentric coordinate with $\mathrm{HD}$, has been introduced to address the affine transformed point pattern matching [14, but the barycentric coordinate relies on the convex shape of points and its mean position. Therefore, if the convex shape is deformed by an outlier, result is not reliable.

Since Euclidean distance of point features is not always sufficient for matching, some methods utilise contextual conditions derived from the point pattern.These methods structure points as a tree or a graph, and use the structure to solve the general PPM problems. In particular, weighted connection of nodes in a graph indicates the strength of connection and this concept is utilised by stochastic approaches, e.g., probabilitic relaxation methods [15. Li et al. introduced a tree structure which is obtained by applying $k$-D tree algorithm to partition points, and the similarity of trees is measured for sparsely distributed point patterns [16]. Strickland et al. suggested a method that uses an iterative relaxation algorithm based on the probability defined by a separation of length and angle between connected branches in a non-rigid shape [1. Zheng et al. considered point matching as an optimisation problem in order to preserve local neighbourhood structure, and the optimal solution is searched using relaxation labelling [3]. Andrew et al. focussed on the Delaunay graph of a set of data points and suggested a relaxation labelling solution for Delaunay graph matching which simplifies the joint probabilities defined by neighbouring nodes and reduces the computation time of the iteration [17. The concept is extended to a transform estimation in an EM framework in their later work [18. Despite these extensive researches, PPM still remains a challenging task: spectral methods are not robust to corruption in structure (e.g., due to noise) and the performance of relaxation methods degrade when there are significant increases in the sizes of the point sets since defining every joint probability is complicated 4 .

In this paper a Delaunay graph is investigated as a method for uniquely constructing a local configuration called a clique, and propose a clique-based HD which exploits a graphical shape difference between cliques. In the proposed method, an idea of robust matching is motivated by HD and the affine invariant features from the cross ratio, and it effectively addresses noise, outliers, occlusion and inexact graph matching. The proposed distance measures inter-distance, i.e., it measures a clique distance directly from a model to a test data unlike a spectral method. Thus, when the sizes of the two graphs being compared are different, a rectangular matrix stores all the possible distances between cliques and strong correspondences are identified to define initial transform between two sets. Once 
the local transform is estimated initially, the guided matching increases point correspondences by collecting point pairs (called supporting pairs) that reside within an error bound of the estimated transform and repeating the estimation.

The paper is organised as follows. A Delaunay tessellation and its characteristics are explained in Sect. 2, which also introduces the theoretical representation of data points used in the proposed method. Section 3 explains how the distance between two cliques is measured and how it is related to HD. Finally, experimental results and conclusions are presented in Sect. 4 and 5, respectively.

\section{Point Set Representation}

A Delaunay graph is a result of Delaunay tessellation. As a dual of a Voronoi diagram which divides distinct $n$ points according to the nearest neighbour rule, the Delaunay graph can be constructed from connecting points of Voronoi polygons that are adjacent to one another [see Fig[1(a) and (b)] and result thus forms a pattern of packed convex polygons [19,20. One characteristic of a Delaunay graph is that no point is allowed within a circumcircle of a triangle. In other words, noise only affects local graph where circumcircles are contaminated [see Fig[1(c)]. Furthermore, even when points are patly occluded, resulting graph is similar to that prat of the original graph (see Fig.1(d)).

Mathematically, the representation of a Delaunay graph is similar to that of an ordinary graph. Suppose that there are point sets of a model $\mathcal{V}^{\mathrm{m}}=$ $\left\{\boldsymbol{v}_{1}, \cdots, \boldsymbol{v}_{\left|\mathcal{V}^{\mathrm{m}}\right|}\right\}$, where $|\cdot|$ is the cardinality of a point set and $\mathcal{V}^{\mathrm{t}}$ is a test

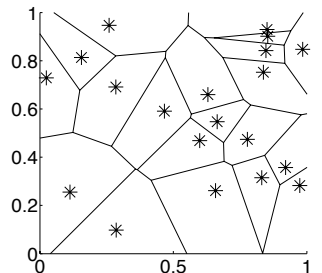

(a)

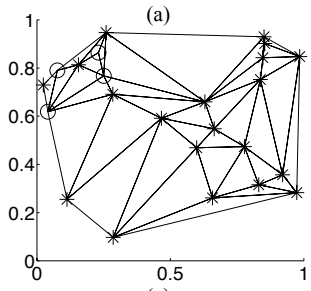

(c)

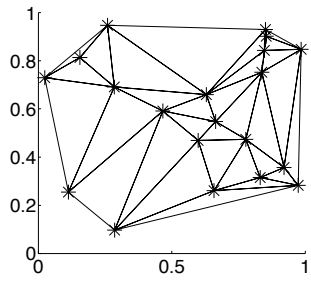

(b)

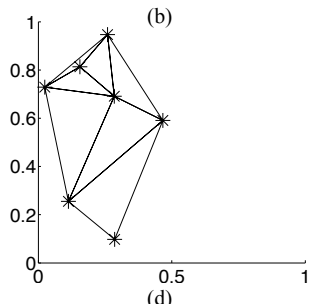

(d)

Fig. 1. (a) A Voronoi diagram of 20 feature points (denoted by '*') that are randomly generated and ranged [0 1]. (b) A Delaunay graph, the dual of the Voronoi diagram in (a). (c) The general shape of Delaunay graph in (b) is not changed by the addition of a noise point 'o'. (d) A randomly selected small portion of features does not change the local graph significantly. 
set. A Delaunay graph is then defined by sets of points, edges and faces, i.e., a graph $\mathcal{G}^{\mathrm{m}}=\left(\mathcal{V}^{\mathrm{m}}, \mathcal{E}^{\mathrm{m}}, \mathcal{F}^{\mathrm{m}}\right)$, where $\mathcal{E}^{\mathrm{m}}$ is the list of node connections (i.e., $\mathcal{E}^{\mathrm{m}}=$ $\left.\left\{(i, j) \mid \forall \boldsymbol{v}_{i}, \boldsymbol{v}_{j} \in \mathcal{V}^{\mathrm{m}}\right\}\right)$, and $\mathcal{F}^{\mathrm{m}}$ is a set of triplets of indices, in which each triplet represents a triangle such as $\mathcal{F}^{\mathrm{m}}=\left\{(i, j, k) \mid \forall(i, j),(j, k)\right.$ and $\left.(j, k) \in \mathcal{E}^{\mathrm{m}}\right\}$. Local entity of a graph called a clique is then defined as a cluster of points connected by $\mathcal{E}^{\mathrm{m}}$. By using clique notation in [17], a model clique centred at a point $\boldsymbol{v}_{i}$ is given by

$$
\mathcal{C}_{i}^{\mathrm{m}}=\{i\} \cup\left\{j \mid \forall(i, j) \in \mathcal{E}^{\mathrm{m}}\right\} .
$$

A centre point $\boldsymbol{v}_{i}$ of a clique $\mathcal{C}_{i}^{\text {m }}$ is especially referred to as the seed of a clique, and the indices of the other points are called neighbours, which are ordered in the clockwise direction.

One benefit of using this structured points is that it creates unique local configuration of points and the connections of nodes are not as complicated as a generic non-directional graphical model, e.g., Markov random fields. This is because the Delauany graph restricts the number of point connections as a triangle and the connections are only made within the nearest neighbours. However, a problem occurs when the sizes of the two cliques being compared are

different, i.e., $\left|\mathcal{C}_{i}^{\mathrm{m}}\right| \neq\left|\mathcal{C}_{j}^{\mathrm{t}}\right|$. Thus, if a general point-pair based distance is used for matching, it is necessary to establish inexact matching of neighbour points in advance, e.g., some points should have multiple opponents, or a null point concept is required [3, 18. However, the proposed matching framework enables distance of two data sets with different sizes to be measured.

\section{Clique Distance}

A similarity transform $H_{s}$ is normally designed with 4 degrees of freedom, i.e., the transform matrix in a $2 \mathrm{D}$ projective space $\mathbb{P}^{2}$ is defined by

$$
H_{s}\left(s, \theta, t_{\mathrm{x}}, t_{\mathrm{y}}\right)=\left[\begin{array}{ccc}
s \cos \theta & s \sin \theta & t_{\mathrm{x}} \\
-s \sin \theta & s \cos \theta & t_{\mathrm{y}} \\
0 & 0 & 1
\end{array}\right] \text {, }
$$

where $s$ is a scaling factor, $\theta$ is a rotational angle, and $t_{\mathrm{x}}$ and $t_{\mathrm{y}}$ represent translations in two orthogonal directions. Therefore, $H_{s}$ describes a rigid motion of an object with a change in scale in 2D space. Some of the useful invariant properties under the similarity transform are that length ratio, the combination of vectors (e.g., centroid), and area ratio are preserved [21. In particular the angle ratio is not affected by this transformation so that the Delaunay graph of a transformed point pattern also remains unchanged. These properties are exploited in a new distance between cliques, which makes the new measure more reliable for a transformed point pattern.

The shape difference of a clique is measured by a graphical distance. Instead of direct use of edge length and angles, the proposed graphical distance compares a set of cross ratios of a clique. The cross ratio is the length ratio defined by four 
distinct points on a collinear line, e.g., the cross ratio of four points, $\boldsymbol{v}_{1}, \boldsymbol{v}_{2}, \boldsymbol{v}_{3}$, and $\boldsymbol{v}_{4}$ is

$$
f_{\mathrm{cr}}\left(\boldsymbol{v}_{1}, \boldsymbol{v}_{2}, \boldsymbol{v}_{3}, \boldsymbol{v}_{4}\right)=\frac{\left\|\boldsymbol{v}_{1}-\boldsymbol{v}_{3}\right\| \cdot\left\|\boldsymbol{v}_{2}-\boldsymbol{v}_{4}\right\|}{\left\|\boldsymbol{v}_{1}-\boldsymbol{v}_{4}\right\| \cdot\left\|\boldsymbol{v}_{2}-\boldsymbol{v}_{3}\right\|} .
$$

Because the cross ratio is invariant up to a projective transform, it can correctly measure the shape difference of two similarity transformed graphs. To retain four points for the cross ratio estimation, the proposed method exploits the midpoints of two sides of a triangle belonging to a clique, and the two ends of the boundary edge. Since these four points are not collinear, the two midpoints are projected onto the boundary line. Suppose a neighbour index of $\mathcal{C}_{i}^{\mathrm{m}}$ is denoted as $n_{i 1}, \cdots, n_{i\left(\left|C_{i}^{\mathrm{m}}\right|-1\right)}$. The boundary of a clique $\mathcal{C}_{i}^{\mathrm{m}}$ is then defined as

$$
\mathcal{B}_{i}^{\mathrm{m}}=\left\{\left(n_{i j}, n_{i(j+1)}\right)|j=1, \cdots,| \mathcal{C}_{i}^{\mathrm{m}} \mid-2\right\} .
$$

If the seed of a clique is enclosed by its neighbours, the boundary set has another element $\left\{\left(n_{i\left(\left|\mathcal{C}_{i}^{\mathrm{m}}\right|-2\right)}, n_{i 1}\right)\right\}$. This is because the face of an internal clique is normally defined by a circular permutation of two adjacent neighbours but a clique whose boundary is identical to the boundary of a graph is not, i.e., the maximum size of a boundary set is limited by $\left|\mathcal{B}_{i}^{\mathrm{m}}\right| \leq\left|\mathcal{C}_{i}^{\mathrm{m}}\right|-1$.

All the midpoints from two side edges of a triangle in $\mathcal{C}_{i}^{\mathrm{m}}$ are stored in

$$
M_{i}^{\mathrm{m}}=\left[\left[\boldsymbol{\mu}_{i 1}^{1} \boldsymbol{\mu}_{i 1}^{2}\right]\left[\boldsymbol{\mu}_{i 2}^{1} \boldsymbol{\mu}_{i 2}^{2}\right] \cdots\left[\boldsymbol{\mu}_{i j}^{1} \boldsymbol{\mu}_{i j}^{2}\right]\right],
$$

where $\boldsymbol{\mu}_{i j}^{1}=0.5\left(\boldsymbol{v}_{i}+\boldsymbol{v}_{n_{i j}}\right)$ and $\boldsymbol{\mu}_{i j}^{2}=0.5\left(\boldsymbol{v}_{i}+\boldsymbol{v}_{n_{i(j+1)}}\right)$. Consequently, each triangle in a clique is represented by the cross ratio. For example, a triangle of $\left(i, n_{i j}, n_{i(j+1)}\right)$ is defined by

$$
r_{i j}=f_{\mathrm{cr}}\left(f_{\mathrm{o}}\left(\boldsymbol{v}_{n_{i j}}, \boldsymbol{\mu}_{i j}^{1^{\prime}}, \boldsymbol{\mu}_{i j}^{2^{\prime}}, \boldsymbol{v}_{n_{i(j+1)}}\right)\right),
$$

where $\boldsymbol{\mu}_{i j}^{k^{\prime}}$ is a projection point of $\boldsymbol{\mu}_{i j}^{k}$ onto the boundary vector $\boldsymbol{v}_{n_{i(j+1)}}-\boldsymbol{v}_{n_{i j}}$, and $f_{\mathrm{o}}(\cdot)$ represents an ordering function which orders four points in the direction of the boundary vector. Figure 2 illustrates some examples of graphical distances of a Delaunay graph from 20 random points. When a triangular face has an obtuse angle, the projections of midpoints lie outside of the triangle [see Fig [2(b)]. In this case, the order of four points is different from the order of the acute triangle shown in Fig. 2(a). To make the order of points consistent, an ordering function $f_{\mathrm{o}}(\cdot)$ rearranges four points in the direction of the boundary vector and any direction of the boundary gives the same cross ratio, i.e., $f_{\text {cr }}\left(\boldsymbol{v}_{1}, \boldsymbol{v}_{2}, \boldsymbol{v}_{3}, \boldsymbol{v}_{4}\right)$ is equal to $f_{\text {cr }}\left(\boldsymbol{v}_{4}, \boldsymbol{v}_{3}, \boldsymbol{v}_{2}, \boldsymbol{v}_{1}\right)$. Thus, the face $\mathcal{F}^{\mathrm{m}}$ can also be represented in terms of the cross ratios of $\mathcal{C}_{i}^{m}$, i.e., $\mathcal{F}^{\mathrm{m}}=\left\{R_{1}^{m}, \cdots, R_{\left|V^{m}\right|}^{m}\right\}$, where $R_{i}^{m}=\left[\begin{array}{lll}r_{i 1} & \cdots & r_{i\left(\left|\mathcal{B}_{i}^{\mathrm{m}}\right|\right)}\end{array}\right]$.

The graphical distance between two cliques is incorporated in the modified $\mathrm{HD}$, where the size of the triangle is used for the weight, i.e.,

$$
h_{\mathrm{g}}\left(R_{k}^{\mathrm{m}}, R_{l}^{\mathrm{t}}\right)=\frac{1}{\left|R_{k}^{\mathrm{m}}\right|} \sum_{\alpha \in R_{k}^{\mathrm{m}}} \min _{\beta \in R_{l}^{\mathrm{t}}}\left\{g\left(r_{k \alpha}, r_{l \beta}\right)\right\},
$$




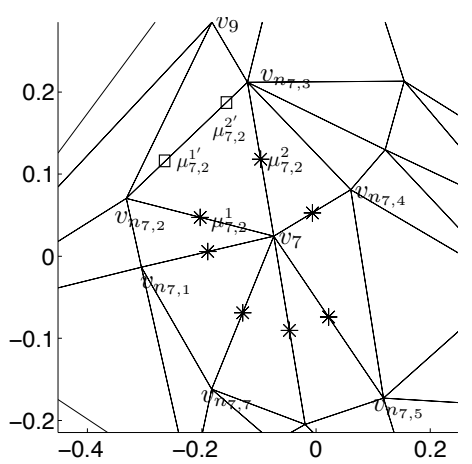

(a)

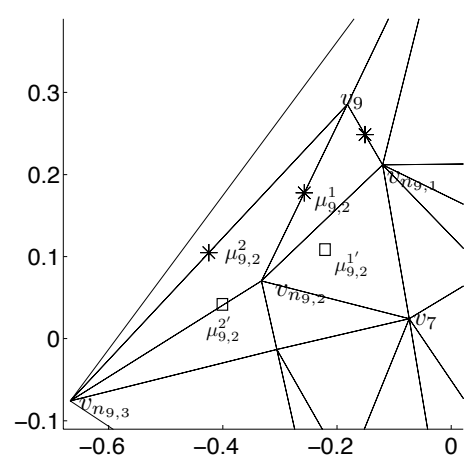

(b)

Fig. 2. Illustration of a graphical distance: (a) A triangle defined by $\left(\boldsymbol{v}_{7}, \boldsymbol{v}_{n_{7,2}}, \boldsymbol{v}_{n_{7,3}}\right)$ is a face of $\mathcal{C}_{7}$ and its cross ratio is $f_{\text {cr }}\left(\boldsymbol{v}_{n_{7,2},}, \boldsymbol{\mu}_{7,2}^{1}, \boldsymbol{\mu}_{7,2}^{2}, \boldsymbol{v}_{n_{7,3}}\right)$; (c) For a face $\left(\boldsymbol{v}_{9}, \boldsymbol{v}_{n_{9,2}}, \boldsymbol{v}_{n_{9,3}}\right)$ with an obtuse angle, the projection of a midpoint is out side of a boundary

where $g(\cdot)$ is

$$
g\left(r_{k \alpha}, r_{l \beta}\right)=\left(1+\left\|\frac{a_{\alpha}}{a_{\mathcal{C}_{k}^{\mathrm{m}}}}-\frac{a_{\beta}}{a_{\mathcal{C}_{l}^{\mathrm{t}}}}\right\|\right)\left\|r_{k \alpha}-r_{l \beta}\right\|,
$$

and $a_{\mathcal{C}_{k}^{\mathrm{m}}}$ is the total area of a clique $\mathcal{C}_{k}^{\mathrm{m}}$, and $a_{\alpha}$ represents the area of a triangle indexed by $\alpha$. Thus, the graphical distance accounts for the difference of area ratio in a clique. The non-directional version of the graphical distance $h_{2}\left(R_{k}^{\mathrm{m}}, R_{l}^{\mathrm{t}}\right)$ is obtained by choosing the maximum of two directional distances. Finally, a graphical proximity matrix is defined as

$$
\Delta_{\mathrm{g}}=\left[\begin{array}{cccc}
h_{2}\left(R_{1}^{\mathrm{m}}, R_{1}^{\mathrm{t}}\right) & h_{2}\left(R_{1}^{\mathrm{m}}, R_{2}^{\mathrm{t}}\right) & \cdots & h_{2}\left(R_{1}^{\mathrm{m}}, R_{\left|\mathcal{V}^{\mathrm{t}}\right|}^{\mathrm{t}}\right) \\
\vdots & \vdots & \cdots & \vdots \\
h_{2}\left(R_{\mid \mathcal{V}^{\mathrm{m} \mid}}^{\mathrm{m}}, C_{1}^{t}\right) & h_{2}\left(R_{\mid \mathcal{V}^{\mathrm{m} \mid}}^{\mathrm{m}}, R_{2}^{\mathrm{t}}\right) & \cdots & h_{2}\left(R_{\left|\mathcal{V}^{\mathrm{m} \mid}\right|}^{\mathrm{m}}, R_{\left|\mathcal{V}^{\mathrm{t}}\right|}^{\mathrm{t}}\right)
\end{array}\right],
$$

and the matrix is normalised by the maximum value of its elements.

\section{Experimental Results}

To evaluate the performance of the proposed method in partial matching, the Harris detector was used to extract 251 model points from a $348 \times 360$ grey level image of a cup. Figure 3 (a) shows the Delaunay graph of the model points overlaid on the image. To create a portion of the model data, a reference line and a sweeping line are used in the experiment. The reference line is parallel to the horizontal axis and has an intersection with the mean point of the model data. The sweeping line sweeps the model points in a clockwise direction at a sweeping angle $\theta_{s}$ measured from the reference line. The sweeping angle determines the size 
of the selected portion. Figure 3(b) shows a selected test data with $\theta_{s}=60^{\circ}$, and its Delaunay graph. Since the general HD is able to cope with partial matching, the performance of HD and its variants in partial matching are compared with that of the clique HD from $\theta_{s}=20^{\circ}$ to $\theta_{s}=340^{\circ}$. The results are shown in Fig[3(c), where matching distances are nomalised for comparison. Despite the small portion of model data selected with $\theta_{s}=60^{\circ}$, the clique HD matching distance of 0.589 is the best, whilst HD, MHD, 30\% Ranked HD (RHD), $50 \%$ RHD and 70\% RHD scores are respectively $0.953,0.786,0.388,0.782$ and 0.783 . This is because the local graphical information is not changed. RHDs score 0 as the size of the portion is close to the model data because they only use the best ranked score. However, this characteristic is not desirable for identification.

Images of three small objects as shown in Fig 4 are used for evaluating the identification capability of the proposed method. Two of the objects have a similar shape except for details of their decoration [see Fig [4(a) and (b)] and the last test image is generated from Fig 4(a) using a similarity transform. The

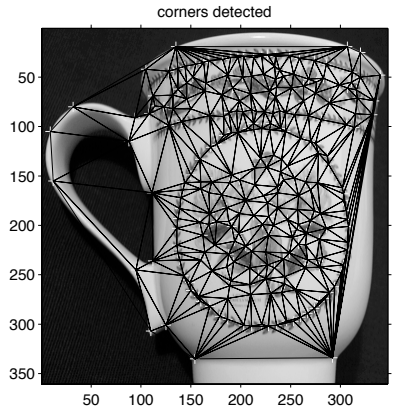

(a)

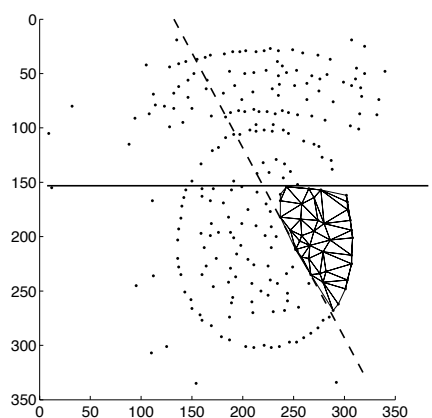

(b)

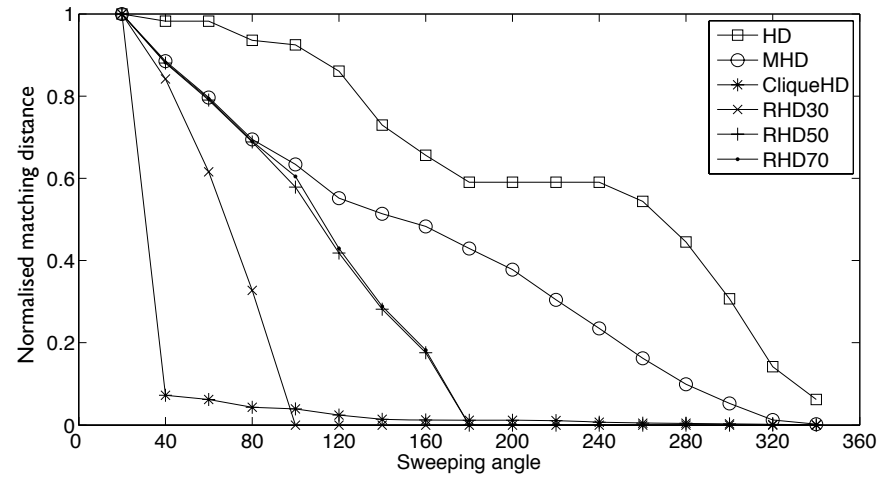

(c)

Fig. 3. (a) The 251 model points are extracted from the $348 \times 360$ image for the partial matching experiment. They are denoted by ${ }^{*}$, with their Delaunay graph overlaid. (b) The selected data is bounded by the solid reference line and the dashed sweeping line with $\theta_{s}=60^{\circ}$. (c) Normalised matching distances of HD, MHD, 30\% RHD, 50\% RHD, $70 \%$ RHD and clique HD, are respectively denoted by $\square, \circ, \mathrm{x},+, \bullet$ and *. 


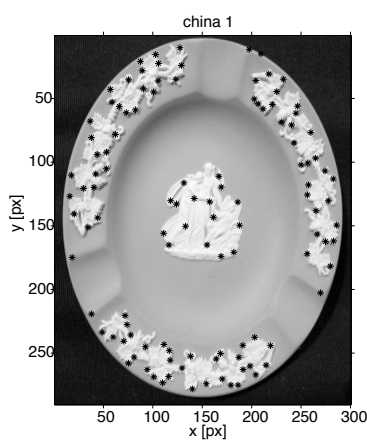

(a)

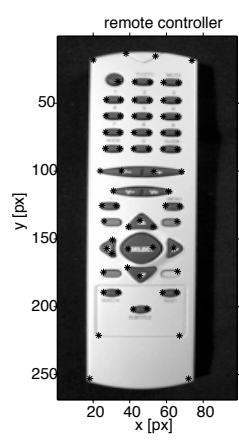

(c)

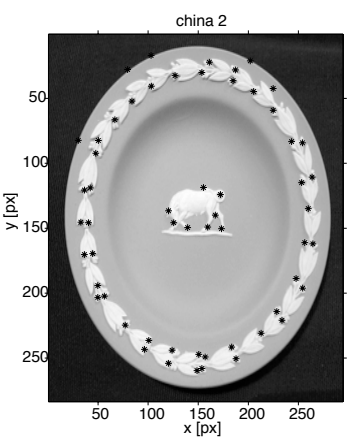

(b)

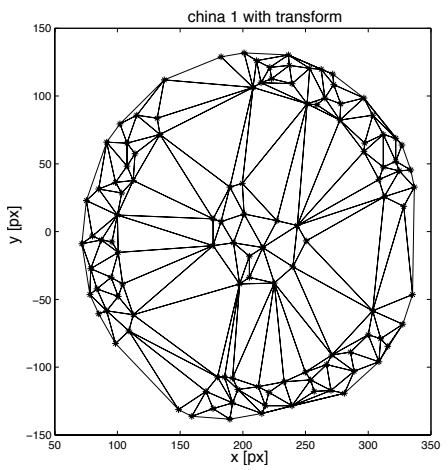

(d)

Fig. 4. Test images for evaluating the identification capability: (a) china 1; (b) china 2 ; (c) remote controller; and (d) image in (a) rotated by $45^{\circ}$ and translated.

images in Fig. 4(a), (b) and (c) respectively generate 113, 57 and 68 feature points.

The normalised matching distances are summarised in Table 1 where a, b, c and d respectively correspond to the objects in Fig. $4(\mathrm{a}),(\mathrm{b}),(\mathrm{c})$ and (d). Since the overall shapes of object (a) and (b) are similar, the HD score (i.e., distance) for these two objects is relatively small. On the other hand, the clique HD gives a larger distance for object (a) and (b) because their local details are different, even though the general shape is not. When a model and test data are related by a similarity transform, clique HD scores 9.83 whereas general HD scores 60.7 (see mataching distance between a and $d$ in Table 1), i.e, the HD matching score between (a) and (d) is significantly increased but clique HD gives a small distance under the transform. These results show that the clique HD achieves a much better performance in identifying an object, e.g., for model based matching. However, when general shape of two objects are considerably different, e.g., rectangular remote and circular china, traditional HD performs better and the average distances of clique HD and HD in this case are 59.73 and 85.21 , respectably (see c column in the table). 
Table 1. Identification test results

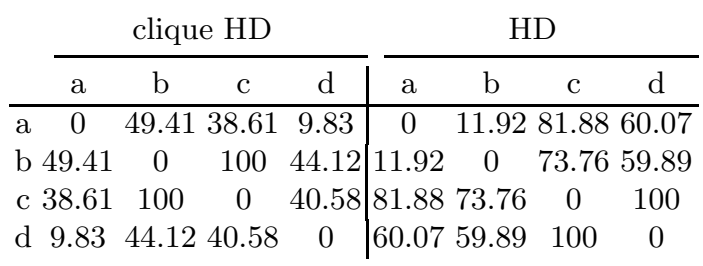

\section{Conclusions}

HD-based point matching has advantageous characteristics, e.g., capable of matching without exact point pairs and partial matching, and robustness against noise and outliers. Therefore, many variants of HD have been introduced. This paper proposes a clique HD that incorporates a graphical distance in the traditional HD matching framework. The proposed method performs matching at local point sets called cliques, which are uniquely formed by the Delaunay tessellation.

To achieve similarity invariance, the proposed method uses a set of cross ratios defined by four collinear points on every boundary edge in a clique. The normalised area of a face in a clique is used for weight when matching two triangles. Experiments show that the proposed matching method is robust to noise and outliers, which normally would deteriorate the performance of graphbased matching methods. However, the method cannot account for apparent shape matching (e.g., matching by the boundary or silhouette of an object) because it is solely based on features from local entities. As future works, the proposed method can be modified to address an affine transformed data, and recent estimating techniques that are robust to noise, e.g., LMeds or RANSAC algorithm will be explored to achieve a more accurate estimation of a local transform.

\section{References}

1. Strickland, R.N., Mao, Z.: Computing correspondences in a sequence of non-rigid shapes. Pattern Recognit. 25(9), 901-912 (1992)

2. Shapiro, L.S., Brady, J.M.: Feature-based correspondence: An eigenvector approach. Image Vision Compt. 10(5), 283-288 (1992)

3. Zheng, Y., Doermann, D.: Robust point matching for nonrigid shapes by preserving local neighborhood structures. IEEE Trans. Pattern Anal. Machine Intell. 28(4), 643-649 (2006)

4. Caetano, T.S., Caelli, T., Schuurmans, D., Barone, D.A.C.: Graphical models and point pattern matching. IEEE Trans. Pattern Anal. Machine Intell. 28(10), 16461663 (2006)

5. Saber, E., Xu, Y., Tekalp, A.M.: Partial shape recognition by sub-matrix matching for partial matching guided image labeling. Pattern Recognit. 38(10), 1560-1573 $(2005)$ 
6. Carcassoni, M., Hancock, E.R.: Spectral correspondence for point pattern matching. Pattern Recognit. 36(1), 192-204 (2003)

7. Caelli, T., Kosinov, S.: An eigenspace projection clustering method for inexact graph matching. IEEE Trans. Pattern Anal. Machine Intell. 26(4), 515-519 (2004)

8. Huttenlocher, D.P., Klanderman, G.A., Rucklidge, W.J.: Comparing images using the hausdorff distance. IEEE Trans. Pattern Anal. Machine Intell. 15(9), 850-863 (1993)

9. Jolly, M.P.D., Jain, A.K.: A modified hausdorff distance for object matching. In: Proc. Int. Conf. Pattern Recognition, vol. A, pp. 566-568 (1994)

10. Yi, X., Camps, O.I.: Line-based recognition using a multidimensional Hausdorff distance. IEEE Trans. Pattern Anal. Machine Intell. 21(9), 901-(1999)

11. Gao, Y., Leung, M.K.H.: Line segment Hausdorff distance on face matching. Pattern Recognit. 35(2), 361-371 (2002)

12. Gao, Y., Leung, M.K.H.: Face recognition using line edge map. IEEE Trans. Pattern Anal. Machine Intell. 24(6), 764-779 (2002)

13. Yu, X., Leung, M.K.H.: Shape recognition using curve segment Hausdorff distance. In: Proc. Int. Conf. Pattern Recognition, vol. 3, pp. 441-444 (2006)

14. Gope, C., Kehtarnavaz, N.: Affine invariant comparison of point-sets using convex hulls and Hausdorff distances. Pattern Recognit. 40(1), 309-320 (2006)

15. Rosenfeld, A., Hummel, R.A., Zucker, S.W.: Scene labeling by relaxation operations. IEEE Trans. Syst., Man, Cybern. 6(6), 420-433 (1976)

16. Baihua Li, Q.m., Holstein, H.: Similarity K-d tree method for sparse point pattern matching with underlying non-rigidity. Pattern Recognit. 38(12), 2391-2399 (2005)

17. Finch, A.M., Wilson, R.C., Hancock, E.R.: Matching Delaunay graphs. Pattern Recognit. 30(1), 123-140 (1997)

18. Cross, A.D.J., Hancock, E.R.: Graph matching with a dual-step EM algorithm. IEEE Trans. Pattern Anal. Machine Intell. 20(11), 1236-1253 (1998)

19. Bowyer, A.: Computing Dirichlet tessellations. Comput. J. 24(2), 162-166 (1981)

20. Aurenhammer, F.: Voronoi diagrams: A survey of a fundamental geometric data structure. ACM Comput. Surv. 23(3), 345-405 (1991)

21. Hartley, R., Zisserman, A.: Multiple view geometry, 1st edn. Cambridge University Press, Cambridge (2000) 